\title{
Generating High-Quality Triangle Meshes from Medical Images
}

\author{
Zhong Chen ${ }^{1}$, Zhiwei Hou ${ }^{1}$, Yuegang Xing ${ }^{2}$ and Xiaobing Chen ${ }^{1}$ \\ ${ }^{1}$ Jiangsu Key Laboratory of Advanced Manufacturing Technology, \\ Huaiyin Institute of Technology, Huaian, 223003, China \\ ${ }^{2}$ Department of Orthodontic Surgery, Huaiyin Hospital of Huaian City, Huaian, \\ 23300, China. \\ zhongchenn19@163.com
}

\begin{abstract}
Due to limited resolution factor, triangle meshes extracted from medical CT images often contain staircase artifacts. In order to remove staircase artifacts during mesh generation and obtain a smooth model from medical images, a novel removing staircase artifacts method is presented. Firstly, we extract contours by using image segmentation and convert a stack of contours into point clouds. Estimating the normals of point clouds and discard outliers by a weighted covariance analysis, and then remove noise by bilateral filtering. Denoising points are fitted by the quadric error function, and then construct triangle meshes by adaptive spherical cover. The resulting triangle meshes are evaluated regarding surface smoothness, volume preservation, geometric accuracy. The experimental results show that the proposed approach can create a smooth, high-quality surface model from medical images.
\end{abstract}

Keywords: Medical CT; point clouds; triangle meshes; staircase artifacts

\section{Introduction}

In medical imaging such as $\mathrm{CT}$ scans, producing anisotropic data in which the slice resolution in the $\mathrm{z}$ direction is commonly lower than the imaging resolution in the $x-y$ plane is possible. In other words, in-plane resolution is only accurate to the pixel, whereas slices can be apart by more than one pixel. A common problem is the appearance of staircase artifacts when triangle meshes from medical images are generated. Usually, medical surface meshes are generated through the marching cubes (MC) algorithm [1] (see Figure 1, in which anisotropic voxel size is $0.352 \times 0.352 \times 0.5 \mathrm{~mm}$ ). Staircase artifacts affect $3 \mathrm{D}$ printing and finite element (FE) simulation. In such applications, smooth, high-quality triangle meshes are essential.

Staircase artifacts can be removed after mesh generation (by mesh smoothing) or during mesh generation (by surface reconstruction). These two methods are described as follows. (1) By mesh smoothing: a variety of mesh smoothing methods have been proposed to improve smoothness. Smoothing methods only allow to reduce staircase artifacts after mesh generation (e.g., Laplacian smoothing [2], Taubin smoothing [3] and Mean Curvature Flow [4]). These methods can remove staircase artifacts, but often cause loss of features and volume shrinkage. Recently, feature-preserving smoothing [5-8] was proposed, these methods can remove the noise while retaining original features. However, staircase artifacts are retained as features when using these feature-preserving smoothing methods. Thus, Moench et al., [9] proposed context-aware mesh smoothing, which can be adaptively smoothed the artifact areas and preserve features in non-artifact areas, but this method was generated many low-quality meshes. Constrained Elastic Surface Nets (CESN) smoothing method [10] reduced staircase artifacts partly but retaining some features, and some obvious staircase may still remain on the surface. (2) By surface 
reconstruction: Implicit surface reconstruction is an active research field in Digital Geometry Processing (DGP). The following methods can generate surface meshes from point clouds directly. Hoppe et al., [11] proposed an implicit reconstruction approach, which produced surface meshes from point clouds by using a signed distance function. Levin [12] and Alexa et al., [13] approximated point clouds with polynomials by moving least squares (MLS). The Multi-level partition of unity implicit was presented by Ohtake et al., [14]. Bade et al., [15] reconstructed vascular surface models by using the MPU method based on point clouds from binary segmentation masks. Zhao et al., [16] constructed surface models from point clouds by level set method [17].

These methods can generate smooth surface, but the staircase artifacts are not entirely removed. Ohtake et al., [18] proposed an integrating approach to meshing point clouds. This method is based on the Garland-Heckbert [19] local quadric error minimization strategy. High-quality meshes can be created from points directly by means of adaptive spherical cover. However, noise and outliers produce a failed surface reconstruction in Ref. [18].



(a) (b)
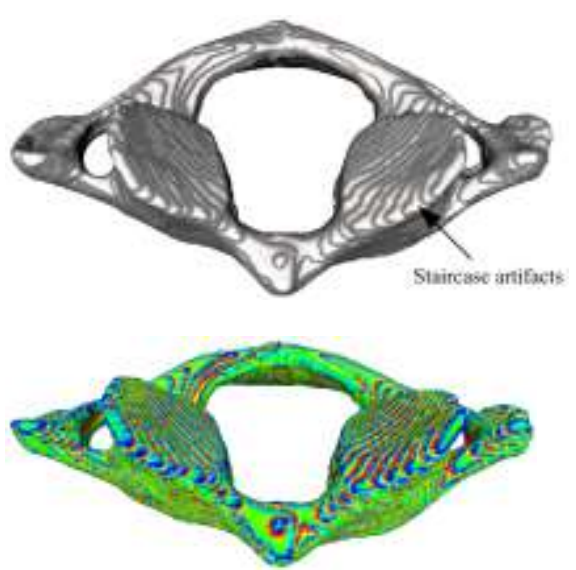

(c)

Figure 1. (a) Medical CT Image Slices of the First Cervical Vertebrae (C1 for Short). (b)Surface Rendering By the Marching Cubes Algorithm. (C) Mean Curvature Visualization

In order to remove staircase artifacts during mesh generation, we have introduced the adaptive spherical cover method into biomedical engineering and modified this method in our work. Our method improves the anti-noise ability and localization accuracy based on the implicit approach.

\subsection{Approach Overview}

An overview of our presented approach to triangle mesh reconstruction is illustrated in Figure 2. The input data derive from medical CT image slices. The process begins with a stack of contours processed by image segmentation (see Section 2.1). We convert a stack of contours into a set of point clouds in a threedimensional space (see Section 2.2). We then construct a data structure of the CT point clouds and query the neighbors by means of the kd-tree. The k-nearest neighbors of medical CT point clouds are constructed and the normals are estimated by means of a weighted covariance analysis (see Section 2.3), and then reduce the noise from point clouds through bilateral filtering (see Section 2.4). Point clouds are fitted by using the quadric error function, and then construct triangle meshes by the adaptive spherical cover (see Section 2.5). 


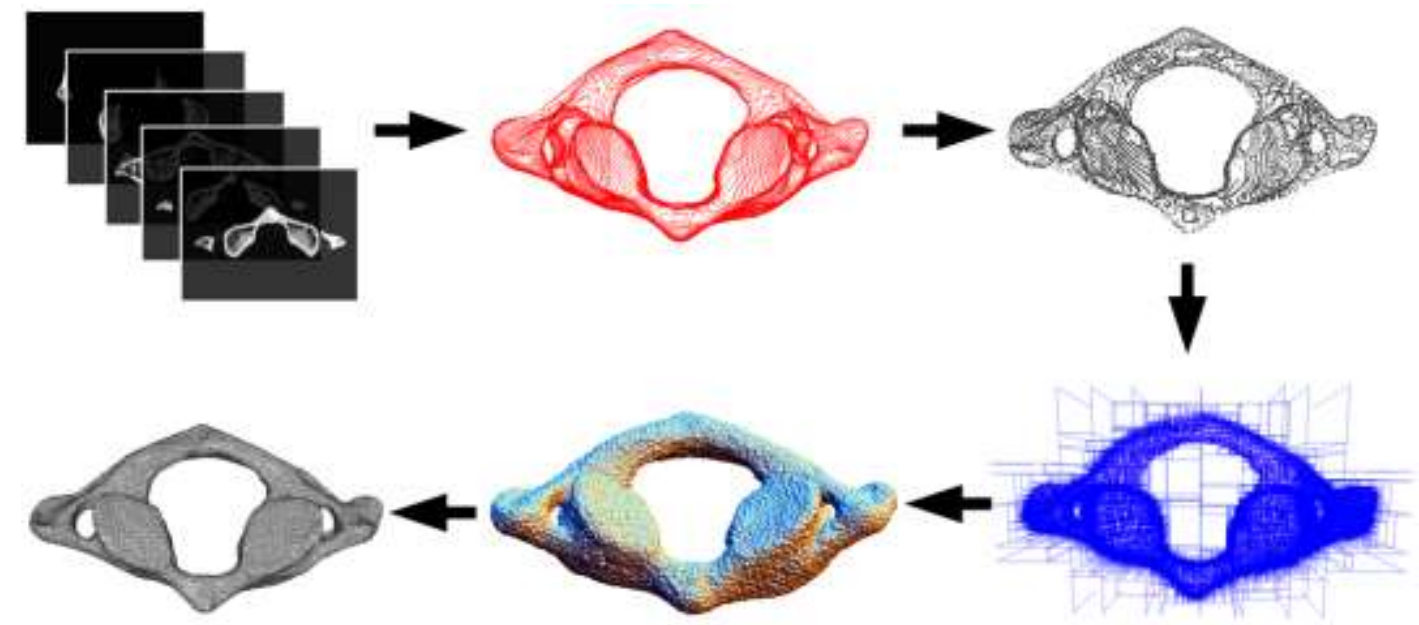

Figure 2. Overview of the Presented Mesh Generation Approach. A Stack of Closed Contours By Image Segmentation. Point Clouds Extract from a Set of Contours. Dividing Point Clouds By Kd-Tree, Estimate the Normals and Reduce the Noise. Constructing Triangle Meshes By the Modified Adaptive Spherical Cover

\section{Surface Reconstruction}

\subsection{Contours Extraction of CT Images}

Medical images used in our study were segmented by an active contour without edges algorithm [20]. The algorithm has certain advantages: (1) it is robust against noise; (2) a closed contour curve by image segmentation under any conditions can be obtained. The main objective of the algorithm is to evolve an interface, which divides the image into two homogeneous regions. This is the solution to minimize the energy functional $F\left(c_{1}, c_{2}, C\right)$, defined by:

$$
\begin{gathered}
F\left(c_{1}, c_{2}, C\right)=\mu \text { Length }(C)+v \text { A rea }(\text { inside } C)+\lambda_{1} \int_{\text {inside }(c)}\left|u(x, y)-c_{1}\right|^{2} d x d y+ \\
\quad \lambda_{2} \int_{\text {outside }(c)}\left|u(x, y)-c_{2}\right|^{2} d x d y
\end{gathered}
$$

where $C$ corresponds to the closed curve, $u(x, y)$ corresponds to the image, $c_{1}$ and $c_{2}$ are the average intensity of $u(x, y)$ inside and outside of $C$, respectively. Additionally, $\mu$, $v \geq 0, \lambda_{1}, \lambda_{2}>0$ are fixed parameters. In this paper, we set the parameters as: $\lambda_{1}=1, \lambda_{2}=7$ (bone) and $\mu=0.1 \times 255^{2}, v=0, \mathrm{~V} t=0.01$ ( $\mathrm{V} t$ is the time step), 120 iterations.

\subsection{Point Clouds Extraction}

To construct bone meshes by means of implicit surface reconstruction, obtaining point clouds of bone images is firstly required. We extract bone contours from CT image sequences and set a single pixel of the precise contour in a $2 \mathrm{D}$ plane as $\mathrm{x}, \mathrm{y}$ coordinates, based on the location in which the profile calculated the $\mathrm{Z}$ coordinate. Finally, we obtain 3D point coordinates and the contours translate into point clouds. Point clouds are only available for 3D coordinate information. No topological information exists and the point cloud of every point of differential geometry information (i.e., normal vector, curvature) can only be calculated through the neighbors of a point. Therefore, establishing topological relations in points is 
necessary and we can easily query the k-nearest neighbors for each point. At present, three k-nearest neighbor queries are commonly used: octree, grid, and kdtree methods [21-22]. The first two methods are classified by a bounding box, and the third approach is to search for the nearest two points. In this study, we adopted the kd-tree method in order to construct the data structure of the CT point clouds and query the neighbors.

\subsection{Normal Estimation}

Point clouds frequently contain noise derived from scanning and transformation processes. CT point clouds that commonly exhibit artifacts of irregular sampling (e.g., noise and outliers) cause difficulties in estimating differential surface attribution. The classical normal estimation is principal component analysis (PCA) [11], which can be unreliable because of outliers and large-scale noise. We describe a novel normal estimation algorithm, which is not affected by unorganized outliers.

CT point clouds $P=\left\{p_{i} \in R^{3}\right\}_{i=1}^{n}$ with $n$ unorganized points, where the $k$ nearest neighbors of an arbitrary point $p_{i}$ is denoted by $\left\{p_{j} \in P\right\}_{j=1}^{k}, k=10$, and $\bar{p}$ is the centroid of the neighbors $\left\{p_{j} \in P\right\}_{j=1}^{k}$. We use a weighted method [23] to estimate the most effective support for a plane and to eliminate the outliers.

We compute a weighted covariance matrix for point $p_{i}$ is given by

$C=\sum_{j=1}^{k}\left(p_{j}-\bar{p}\right)^{\mathrm{T}} \cdot\left(p_{j}-\bar{p}\right) \cdot g_{j}$

$$
\bar{p}=\frac{1}{k} \sum_{j=1}^{k} p_{j}
$$

where $g_{j}=\exp \left(-d_{j}^{2} / \mu^{2}\right)$ is the weight for point $p_{j}$, If point $p_{j}$ is an isolated point, and $g_{j}=1$. If point $p_{j}$ is an inlier point, then $\mu$ is the mean distance from the point $p_{i}$ to all its neighbors $p_{j}$, and $d_{j}$ is the distance from point $p_{i}$ to its neighbor $p_{j}$.

Consider the eigenvector problem

$C \cdot v=\lambda \cdot v$

where $C$ is a symmetric and positive semidefinite, all eigenvalues $\lambda$ are real-valued and the eigenvectors $v$ form an orthogonal frame. The eigenvector that corresponds to the smallest eigenvalue is considered as the normal vector $\boldsymbol{n}_{i}$ of the point $p_{i}$.

\subsection{Noise Removal}

Point clouds extracted from CT images often contain undesirable noise, which can be reduced by means of various filtering techniques, such as low-pass filtering and bilateral filtering. Fleishman et al., [6] have proposed a bilateral filtering method, which was modified the bilateral filtering from image denoising to mesh denoising and achieve satisfactory results. The bilateral filtering is also suitable for point clouds denoising. Furthermore, bilateral filtering can not disturb the original shape and structure. We calculate a new position of a point $p_{i}$ by means of Equations (4) and (5). 
$d=\frac{\sum_{j=1}^{k} W_{c}\left(\left\|p_{i}-p_{j}\right\|\right) W_{s}\left(\left\|\left\langle p_{i}-p_{j}, \boldsymbol{n}_{i}\right\rangle\right\|\right)\left\langle p_{i}-p_{j}, \boldsymbol{n}_{i}\right\rangle}{\sum_{j=1}^{k} W_{c}\left(\left\|p_{i}-p_{j}\right\|\right) W_{s}\left(\left\|\left\langle p_{i}-p_{j}, \boldsymbol{n}_{i}\right\rangle\right\|\right)}$

$W_{c}(x)=\exp \left(-x^{2} / 2 \sigma_{c}^{2}\right)$

$W_{s}(x)=\exp \left(-x^{2} / 2 \sigma_{s}^{2}\right)$

where $d$ is a signed distance, $W_{c}(x)=\exp \left(-x^{2} / 2 \sigma_{c}^{2}\right)$ is a spatial weighting function, $W_{s}(x)=\exp \left(-x^{2} / 2 \sigma_{s}^{2}\right)$ is a feature-preserving weighting function, $\left\|\boldsymbol{p}_{i}-\boldsymbol{p}_{j}\right\|$ is the distance from a point $\boldsymbol{p}_{i}$ to a neighbor $\boldsymbol{p}_{j},\left\langle\boldsymbol{p}_{i}-\boldsymbol{p}_{j}, \boldsymbol{n}_{i}\right\rangle$ is a vector product operator of both distance vector $\boldsymbol{p}_{i}-\boldsymbol{p}_{j}$ and vector $\boldsymbol{n}_{i}$ of the point $\boldsymbol{p}_{i}, \sigma$ is the standard deviation, and $k$ is nearest neighbors of $\boldsymbol{p}_{i}$.We set $\sigma_{c}=3$ and $\sigma_{s}=2$.

Thus we obtain the following point update equation:

$p_{i}^{\prime}=p_{i}+d \boldsymbol{n}_{i}$

where $p_{i}^{\prime}$ is a new point after bilateral filtering, and $\boldsymbol{n}_{i}$ is a normal vector at a point $p_{i}$.

Finally, we get a new point clouds $P^{\prime}=\left\{p_{i}^{\prime} \in R^{3}\right\}_{i=1}^{l} \quad(l \leq n)$ after bilateral filtering.

\subsection{Spherical Cover}

After noise removal, we construct triangle meshes by linking the auxiliary points in the spheres [18]. This algorithm will generates $m$ spheres centered at $c=\left\{c_{1}, \cdots, c_{m}\right\} \in P^{\prime}$ and the radii of the spheres $r=\left\{r_{1}, \cdots, r_{m}\right\}$ are chosen adaptively. We assign the weight $w_{i}$ to the point $p_{i}^{\prime}$. The function $Q(c, r, x)$ computes a weighted sum of the squared distances from point $x$ to the tangent planes at $\left\{p_{j}^{\prime} \in P^{\prime}\right\}_{j=1}^{k}$ within the spherical region $\left\|p_{j}^{\prime}-c\right\| \leq R$, and $R=T_{q} r$, where $T_{q}$ is a user specified parameter.

$Q(c, r, x)=\sum_{j} w_{j} G_{\sigma}\left(\left\|p_{j}^{\prime}-c\right\|\right)\left(\boldsymbol{n}_{j}^{\prime} \cdot\left(x-p_{j}^{\prime}\right)\right)^{2}$

$w_{i}=\frac{1}{k} \sum_{j=1}^{k}\left\|p_{i}^{\prime}-p_{j}^{\prime}\right\|^{2}$

where $G_{\sigma}(\rho)$ is a Gaussian-like function. If $r$ is fixed, point $x_{\min }=x_{\min }(r)$, the minimizer of $Q(c, r, x)$ is easily found by solving equations.

$E(r)=\frac{1}{L} \sqrt{Q\left(c, r, x_{\min }\right)} \quad E(r)=T_{\text {err }}$

where $E(r)$ is an error function, $L$ is the length of a main diagonal of the bounding box of the point clouds $P^{\prime}=\left\{p_{i}^{\prime} \in R^{3}\right\}_{i=1}^{l}$, and $T_{e r r}$ is an error control threshold.

Once $r$ is fixed, we determine whether the point $x_{\min }$ lies inside or outside of $\|x-c\| \leq r$. If $x_{\min }$ is within this region, we use $x_{\min }$ as the auxiliary point. Otherwise, we 
use $c$ as the auxiliary point. After the adaptive spherical cover, we can get triangle meshes by connecting auxiliary points together.

\section{Experimental Results and Discussion}

\subsection{Comparison with Other Methods}

In this section, we discuss the results of our experiments with several algorithms. We evaluated our approach based on several datasets that include human C1 and Scapula. Medical CT image slices of C1, as shown in Figure 1a, with an anisotropic voxel size of $0.352 \mathrm{~mm} \times 0.352 \mathrm{~mm} \times 0.5 \mathrm{~mm}$, In other words, slice distance was $0.5 \mathrm{~mm}$. The slice distance was considerably larger than the in-plane resolution of $0.352 \mathrm{~mm} \times 0.352 \mathrm{~mm}$. The voxel size of the scapula was $0.352 \mathrm{~mm} \times 0.352 \mathrm{~mm} \times 1 \mathrm{~mm}$. These medical surface meshes were generated by using the marching cubes algorithm (see Figure 3). The MC triangle meshes as reference models for evaluation of geometric parameters changes in this paper, detailed parameters as shown in Table 1 . We can see staircase artifacts on the $\mathrm{C} 1$ and scapula surface derived from marching cubes (see Figure 3). The resulting triangle meshes were compared with respect to surface smoothness, volume preservation and geometric accuracy. All of the results are displayed using mean curvature visualization (see the bottom of Figure 3, Figure 4, and Figure 5), which can improve the apparent smoothness of a model.
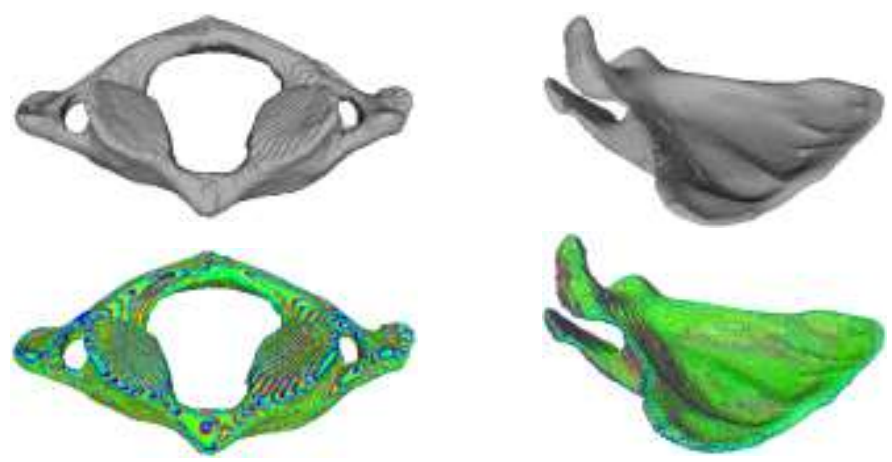

\section{Figure 3. Extracted Surface Mesh Model from CT Images by Using Marching Cubes}

Comparisons with other methods are based on the removal of the staircase artifacts. The best results of the above methods are the maximum removal of the staircase artifacts. Fleishman's method [6] is successful in preserving original features. After 2 iterations of the method, it is possible to get the best results. However, staircase artifacts would be preserved as the feature with this method, as shown in Figure 4a, and Figure 5a. For the C1 mesh model, after 6 iterations can remove part of staircase artifacts by Laplacian smoothing method. After 19 iterations, all staircase artifacts are removed, but the deformation of the mesh model is very serious. Thus, this method is not suitable for clinical application. From 6 to 19 iterations of this method can be conducted in experiments without damage to the original model structure. Eleven iterations achieve the best results, as shown in Figure 4b. Similarly, for the scapula mesh model, we can achieve the best results after 17 iterations (see Figure $5 b$ ). The main content of our approach is as follows: In a first step, a set of closed contours by image segmentation (for segmentation parameters: see Section 2.1). Then, we have translated closed contours into point clouds. After noise removal (with denoising parameters: $\sigma_{c}=3, \sigma_{s}=2$, and 3 iterations). We construct $\mathrm{C} 1$ and Scapula meshes by linking the auxiliary points in the spheres. For the 
C1 data, we set to $T_{e r r}=1.0 \times 10^{-5}$ and $T_{q}=3$. For the Scapula data, we set to $T_{\text {err }}=1.0 \times 10^{-5}$ and $T_{q}=2$, to account for the large slice distance. In Figure 4c and Figure $5 c$, our approach can efficiently remove staircase artifacts, while preserving the original model structure accurately.

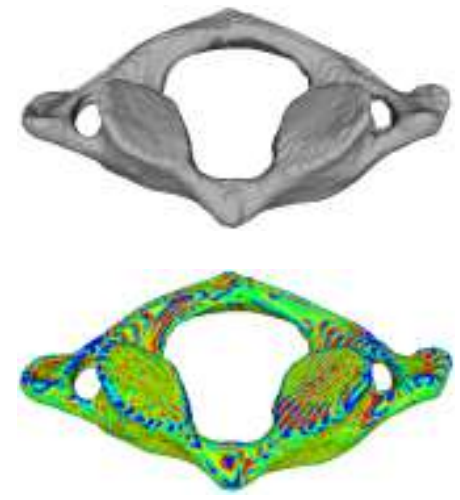

(a)
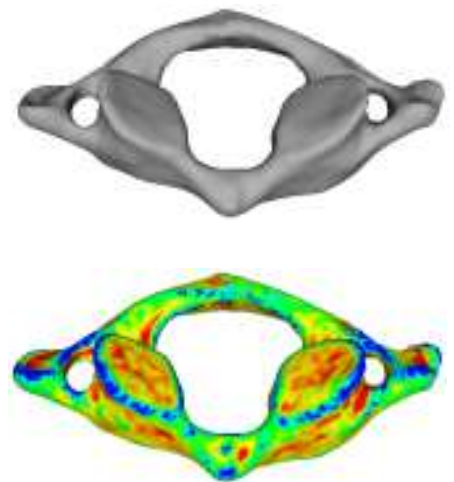

(b)
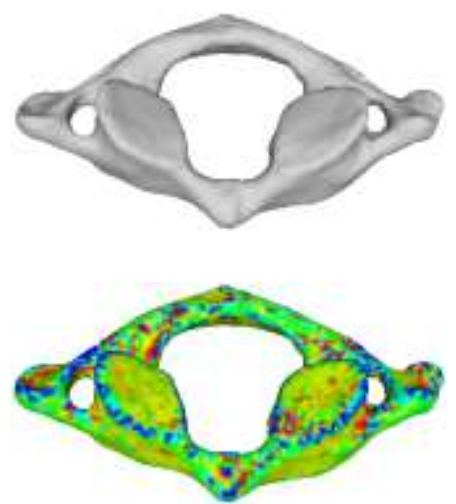

(c)

Figure 4. Comparisons of Results on C1 Model. (a) Smoothing Reference Model By Fleishman's Method.(b) Smoothing Reference Model By Laplacian Method. (c) Surface Mesh Reconstruction From Point Clouds By Our Approach
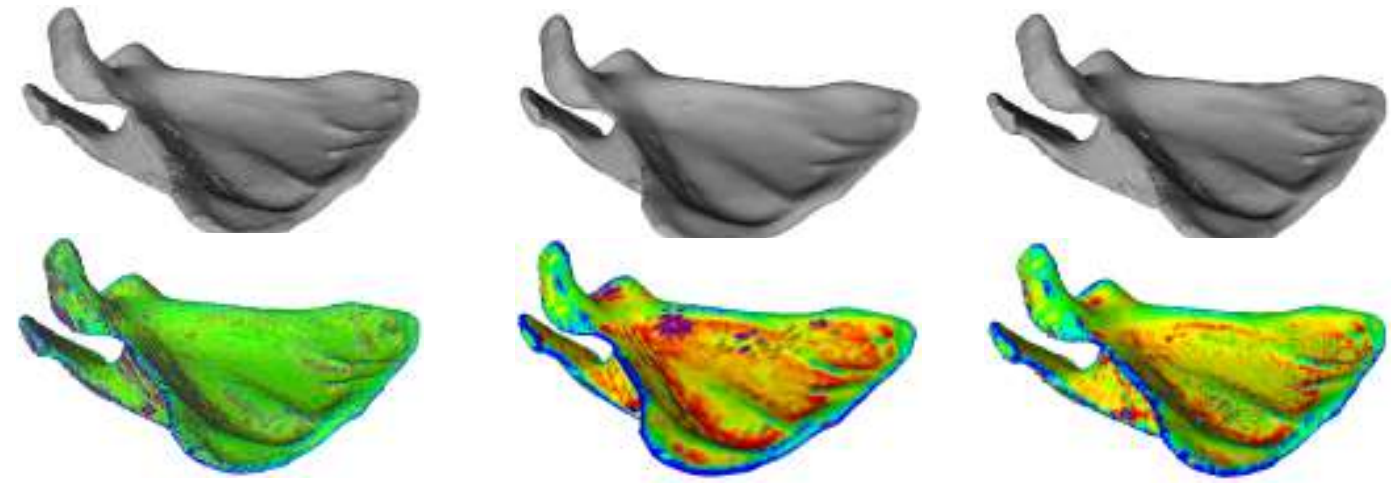

Figure 5. Comparison of Results of Scapula Model. (a) Smoothing Reference Model By Fleishman's Method.(b) Smoothing Reference Model By Laplacian Method. (c) Surface Mesh Reconstruction from Point Clouds By Our Approach

The Hausdorff distance is used to measure quantitatively the difference between the triangle meshes for each object. In our work, the Hausdorff distance is computed from the processed surface to the initial MC reference model. Three types of errors are calculated: maximum, mean and root mean square (RMS). The numerical results as presented in Table 2. It shows that the accuracy of our approach outperforms the other two methods. Table 2, also reveals that the Laplacian smoothing produces obvious volume shrinkage. Our algorithm can retain some level of volume. The error regarding distance changes can also be reduced by using our method with Laplacian smoothing or bilateral filtering algorithm. In general, our method achieved the smallest error compared to the other two smoothing methods. 
Table 1. Characteristics of Initial MC Reference Model

\begin{tabular}{cccccc}
\hline Model & Triangles & Vertices & $\begin{array}{c}\text { Bounding box } \\
(\mathbf{m m})\end{array}$ & $\begin{array}{c}\text { Surface } \\
\left(\mathbf{m m}^{\mathbf{2}}\right)\end{array}$ & $\begin{array}{c}\text { Volume } \\
\left(\mathbf{m m}^{\mathbf{3}}\right)\end{array}$ \\
\hline $\mathrm{C} 1$ & 100222 & 50103 & $75.93 \times 42.32 \times 22.49$ & 5104.57 & 9991.69 \\
Scapula & 354728 & 177361 & $149.50 \times 127.62 \times 66.74$ & 29688.83 & 59442.46 \\
\hline
\end{tabular}

Table 2. Results for the Comparison of Removing Staircase Artifacts Methods

\begin{tabular}{cccccccc}
\hline Model & Method & $\begin{array}{c}\text { Bounding box } \\
(\mathbf{m m})\end{array}$ & $\begin{array}{c}\text { Surface } \\
\left(\mathbf{m m}^{2}\right)\end{array}$ & $\begin{array}{c}\text { Volume } \\
\left(\mathbf{m m}^{\mathbf{3}}\right)\end{array}$ & $\begin{array}{c}\text { Max } \\
\text { error } \\
(\mathbf{m m})\end{array}$ & $\begin{array}{l}\text { Mean } \\
\mathbf{e r r o r} \\
(\mathbf{m m})\end{array}$ & $\begin{array}{l}\text { RMS } \\
(\mathbf{m m})\end{array}$ \\
\hline \multirow{2}{*}{$\mathrm{C} 1$} & $\begin{array}{c}\text { Fleishman's } \\
\text { approach }\end{array}$ & $75.84 \times 42.20 \times 22.49$ & 4997.04 & 9968.63 & 0.229148 & 0.020191 & 0.02646 \\
& $\begin{array}{c}\text { Laplacian } \\
\text { Our } \\
\text { approach }\end{array}$ & $75.52 \times 41.92 \times 22.21$ & 4792.56 & 9795.60 & 0.429040 & 0.075004 & 0.098976 \\
& $75.91 \times 42.27 \times 22.53$ & 5053.23 & 9999.92 & 0.166270 & 0.012789 & 0.016649 \\
\multirow{2}{*}{ Scapula } & $\begin{array}{c}\text { Fleishman's } \\
\text { approach }\end{array}$ & $149.49 \times 127.43 \times 66.60$ & 28888.35 & 59295.07 & 0.452441 & 0.036528 & 0.048803 \\
& $\begin{array}{c}\text { Laplacian } \\
\text { Our } \\
\text { approach }\end{array}$ & $149.13 \times 126.78 \times 66.20$ & 28096.31 & 58431.19 & 0.746007 & 0.105434 & 0.146399 \\
& $149.57 \times 127.60 \times 66.75$ & 29309.90 & 59464.30 & 0.298204 & 0.018302 & 0.024012 \\
\hline
\end{tabular}

\subsection{Mesh Quality}

Generating triangular meshes of high-quality is important to the quality of the volume tetrahedral mesh and the convergence of finite element analysis. In order to evaluate the triangular mesh quality, we compute the radius ratio of the triangles, which is defined as:

$\rho=\frac{2 r_{\text {in }}}{r_{\text {circ }}}$

where $r_{i n}$ is the radius of the inscribed circle of the triangle, $r_{\text {circ }}$ is the radius of the circumscribed circle of the triangle, as shown in Figure 6. With this definition, the equilateral triangle has $\rho=1$ and the flat triangle tends to infinitesimal.

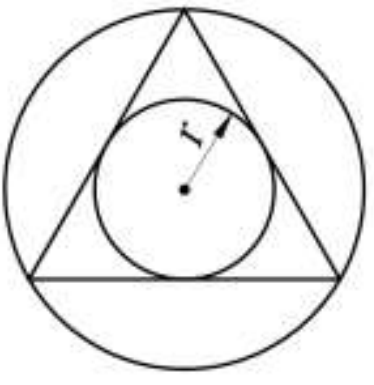

(a)

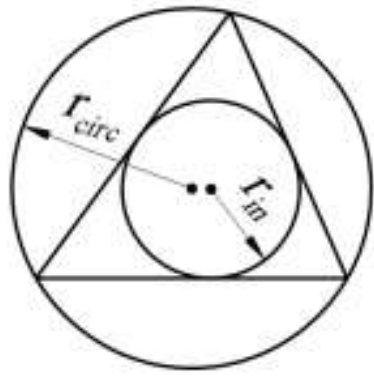

(b)

Figure 6. The Triangle Radius Ratio between the Inscribed and the Circumcircle. (a) Equilateral Triangle with $r_{i n}=r, \quad r_{\text {circ }}=2 r$; (b) For a Triangle, $\rho=2 r_{\text {in }} / r_{\text {circ }}$.

The statistical distribution of mesh quality is shown from Figure 7, to Figure 10 . Because of the triangular mesh density has achieved the voxel level by MC algorithm, the 
number of meshes is very large (see Table 1). So that the colour of these wireframe models look like more black (see Figure 7, and Figure 9,). Besides, these meshes contain many narrow triangles. There are a few of meshes, which radius ratio is close to 1 . Because of putting the triangle connecting three auxiliary points which instead of the all points of inside the convex hull by spherical cover method, we can get sparse and adaptive meshes. As mentioned above, $\mathrm{C} 1$ model has 27231 vertices and 54478 triangles by our approach, Scapula model has 31355 vertices and 62714 triangles. Even more important, there are most of meshes, which radius ratio is close to 1 by our approach (see Figure 8, and Figure 10). These statistical figures demonstrate that the proposed approach can create high-quality surface meshes from medical CT images.

Triangles

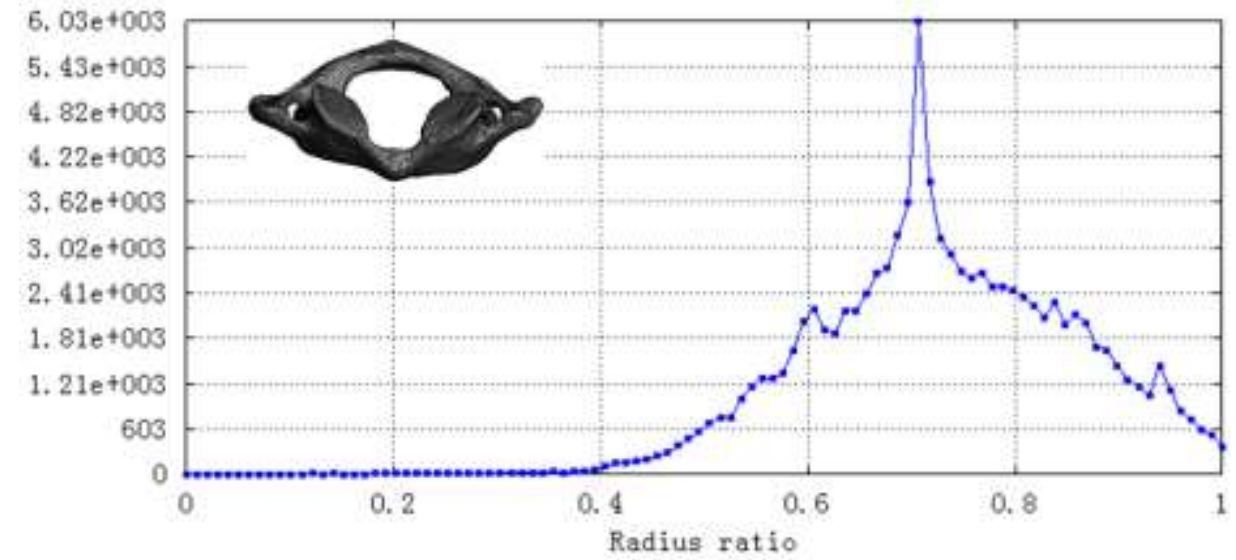

Figure 7. Mesh Quality Statistic of C1 Model by MC Algorithm

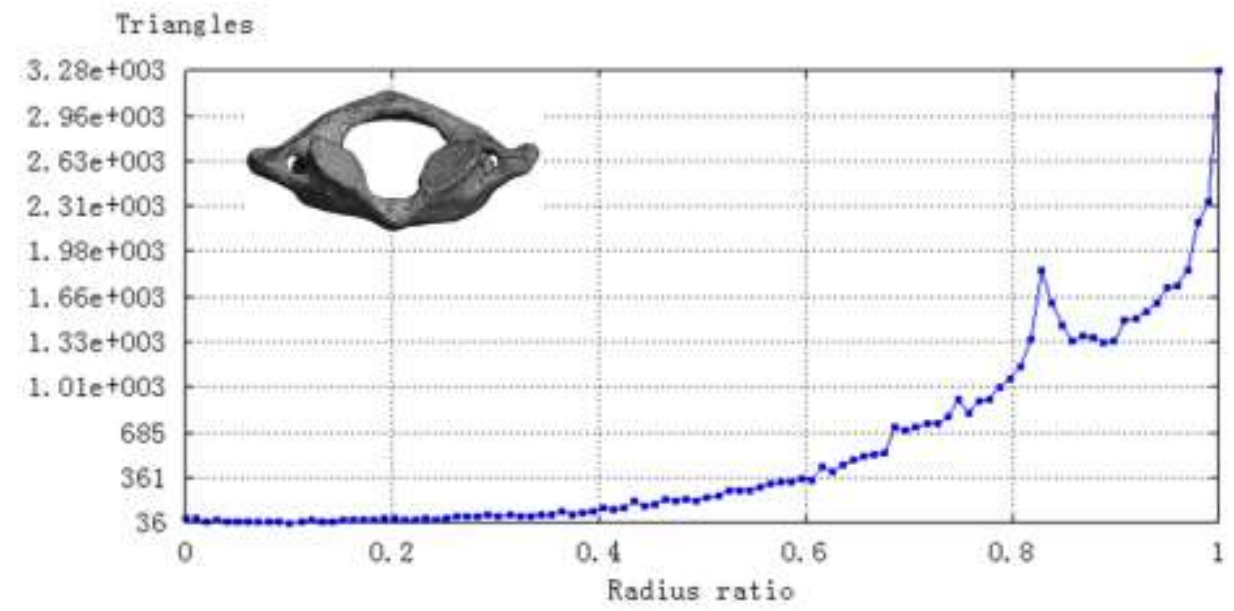

Figure 8. Mesh Quality Statistic of C1 Model by Our Approach 


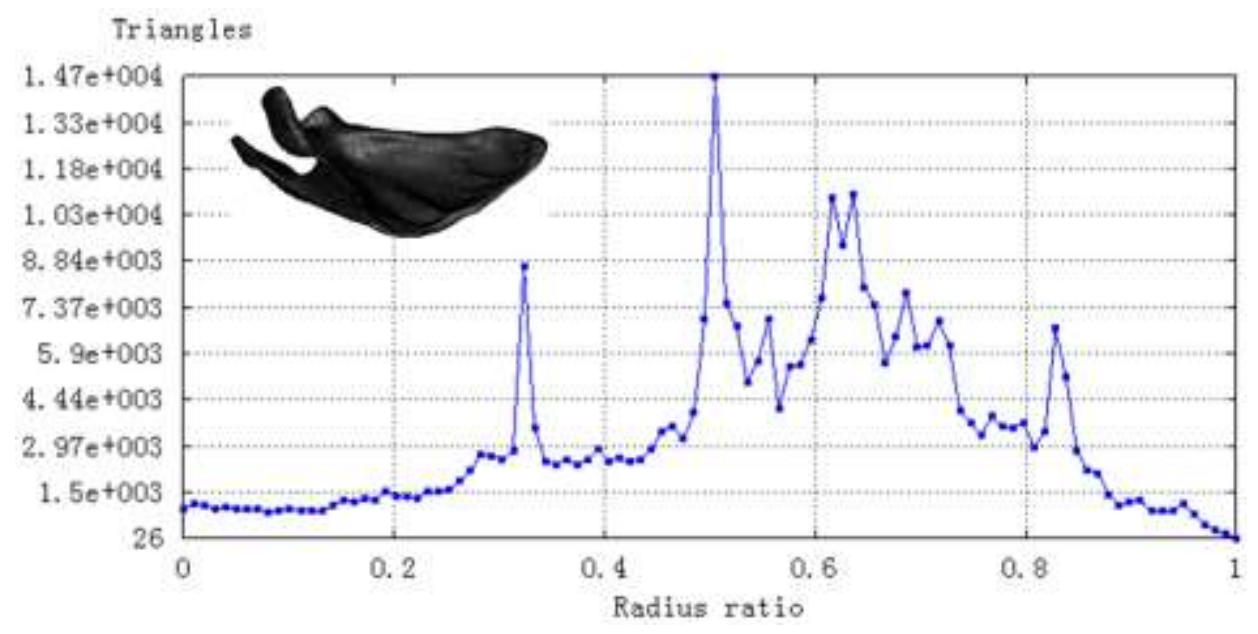

Figure 9. Mesh Quality Statistic of Scapula Model by MC Algorithm

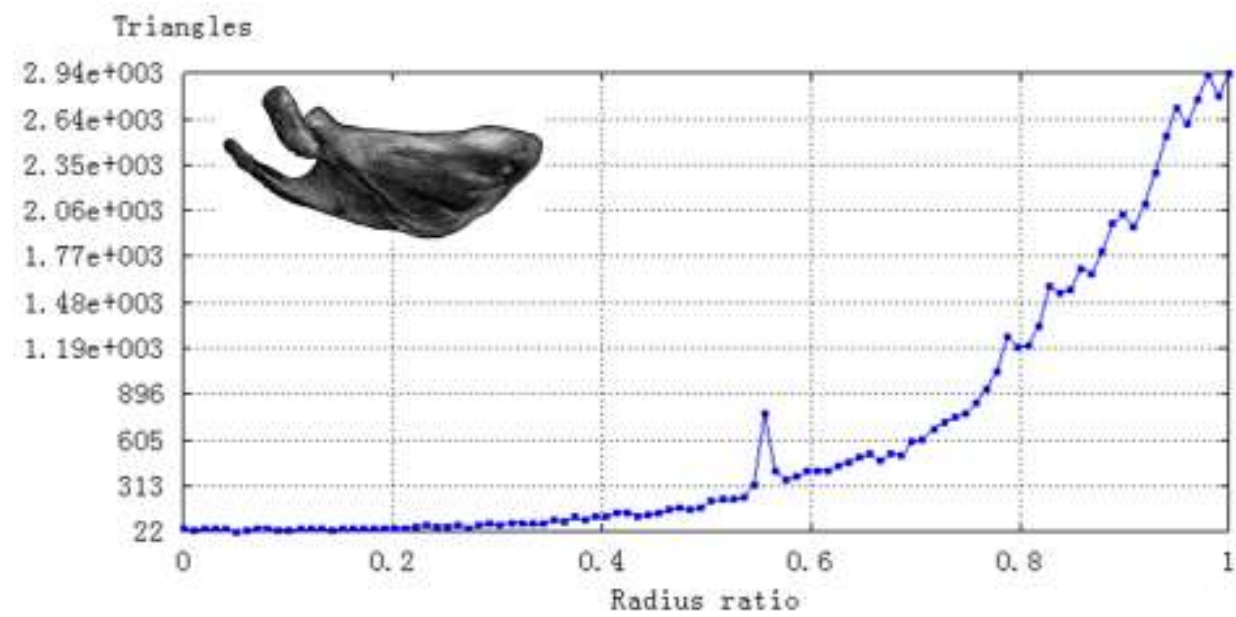

Figure 10. Mesh Quality Statistic of Scapula Model by Our Approach

\section{Conclusion}

In this study, we presented a novel method that removes staircase artifacts and creates smooth surface meshes from medical images. This is a multi-stage process. First, we extract a stack of contours by means of image segmentation. The contours translate into point clouds. We then use a weighted covariance analysis to estimate normal at each point. A bilateral filtering is applied to remove noise from point clouds. Triangle meshes are then constructed using an improved adaptive spherical cover algorithm. Experimental results show that the proposed method can efficiently remove staircase artifacts and generate high-quality triangle meshes with reasonable accuracy.

\section{Acknowledgments}

This work is supported by the Natural Science Foundation of Jiangsu Higher Education Institutions (No.14KJB460002) and (No.15KJA460003), Huaian Scientific and Technological Key Project (No.HAG2015032), Jiangsu Province Industry-UniversityResearch Cooperation Prospective Study Project (No.BY2016061-14), and Jiangsu Key Laboratory of Advanced Manufacturing Technology (No.HGDML-1204). 


\section{References}

[1] W. E. Lorensen and H. E. Cline, "Marching cubes: A high resolution 3D surface construction algorithm", ACM SIGGRAPH computer graphics, (1987), pp. 163-169.

[2] A. Nealen, T. Igarashi, O. Sorkine and M. Alexa, "Laplacian mesh optimization", Proceedings of the 4th international conference on Computer graphics and interactive techniques in Australasia and Southeast Asia, (2006), pp. 381-389.

[3] G. Taubin, "A signal processing approach to fair surface design", Proceedings of the 22nd annual conference on Computer graphics and interactive techniques, (1995), pp. 351-358.

[4] M. Desbrun, M. Meyer, P. Schröder and A. H. Barr, "Implicit fairing of irregular meshes using diffusion and curvature flow", Proceedings of the 26th annual conference on Computer graphics and interactive techniques, (1999), pp. 317-324.

[5] T. R. Jones, F. Durand and M. Desbrun, "Non-iterative feature-preserving mesh smoothing", ACM Transactions on Graphics (TOG), (2003), pp. 943-949.

[6] S. Fleishman, I. Drori and D. Cohen-Or, "Bilateral meshes denoising", ACM Transactions on Graphics (TOG), (2003), pp. 950-953.

[7] T. Tasdizen and R. Whitaker, "Anisotropic diffusion of surface normals for feature preserving surface reconstruction", Proceedings of the fourth International Conference on 3-D Digital Imaging and Modeling, (2003), pp. 353-360.

[8] J. Digne, D. Cohen-Steiner, P. Alliez, F. De Goes and M. Desbrun, "Feature-preserving surface reconstruction and simplification from defect-laden point sets", Journal of mathematical imaging and vision, vol. 48, no. 2, (2014), pp. 369-382.

[9] T. Moench, R. Gasteiger, G. Janiga, H. Theisel and B. Preim, "Context-aware mesh smoothing for biomedical applications", Computers \& Graphics, vol. 35, no. 4, (2011), pp. 755-767.

[10] S. F. F. Gibson, "Constrained elastic surface nets: Generating smooth surfaces from binary segmented data", Medical Image Computing and Computer-Assisted Interventation-MICCAI'98. Springer Berlin Heidelberg, (1998), pp. 888-898.

[11] H. Hoppe, T. DeRose, T. Duchamp, J. McDonald and W. Stuetzle, "Surface reconstruction from unorganized points", Proceedings of SIGGRAPH, (1992), pp. 71-78.

[12] D. Levin, "The approximation power of moving least-squares", Mathematics of Computation, vol. 67, no. 224, (1998), pp. 1517-1531.

[13] M. Alexa, J. Behr, D. Cohen-Or, S. Fleishman, D. Levin and C.T. Silva, "Computing and rendering point set surfaces", IEEE Transactions on Visualization and Computer Graphics, vol. 9, no. 1, (2003), pp. 3-15.

[14] Y. Ohtake, A. Belyaev, M. Alexa, G. Turk and H. P. Seidel, "Multi-level partition of unity implicits", Proceedings of SIGGRAPH, (2003), pp. 463-470.

[15] R. Bade, C. Schumann, S. Seshadhri, G. Janiga, T. Bölke and O. Krischek, "High-quality surface generation for flow simulation in cerebral aneurysms", CURAC, (2007), pp. 125-128.

[16] H. K. Zhao, S. Osher and R. Fedkiw, "Fast surface reconstruction using the level set method", Proceedings of the IEEE Workshop on Variational and Level Set Methods in Computer Vision, (2001), pp. 194-201.

[17] S. Osher and R. Fedkiw, "Level Set Methods and Dynamic Implicit Surfaces", Springer, (2006).

[18] Y. Ohtake, A. Belyaev and H. P. Seidel, "An integrating approach to meshing scattered point data", Proceedings of the 2005 ACM Symposium on Solid and Physical Modeling, (2005), pp. 61-69.

[19] M. Garland and P. S. Heckbert, "Surface simplification using quadric error metrics", Proceedings of the 24th annual conference on Computer graphics and interactive techniques, (1997), pp. 209-216.

[20] T. F. Chan and L. Vese, "Active contours without edges", IEEE Transactions on Image Processing, vol. 10, no. 2, (2001), pp. 266-277.

[21] S. Rusinkiewicz and M. Levoy, "QSplat: A ultiresolution point rendering system for large meshes", Proceedings of the 27th annual conference on Computer graphics and interactive techniques, (2000), pp. 343-352.

[22] C. Dachsbacher, C. Vogelgsang and M. Stamminger, "Sequential point trees", ACM Transactions on Graphics (TOG), (2003), pp. 657-662.

[23] R. B. Rusu, Z. C. Marton, N. Blodow, M. Dolha and M. Beetz, "Towards 3D point cloud based object maps for household environments", Robotics and Autonomous Systems, vol. 56, no. 11, (2008), pp. 927-941. 


\section{Authors}

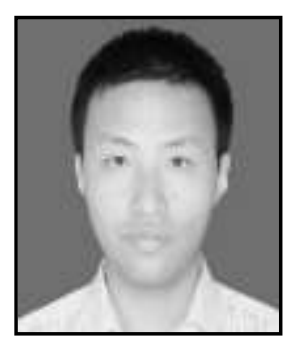

Zhong Chen, He was born in 1982. He received the Ph.D. degree from Chongqing University in 2013. Currently, he is a lecturer in Huaiyin Institute of Technology, China. His research interests include reverse engineering and digital geometry processing.

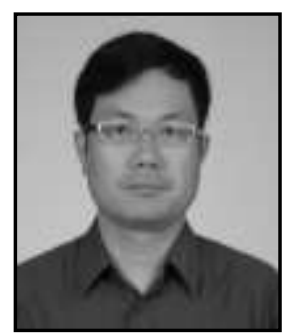

Zhiwei Hou, He was born in 1966. He received the Ph.D. degree from Nanjing University of Aeronautics and Astronautics in 2014.Currently, he is a professor in Huaiyin Institute of Technology, China. His research interests include reverse engineering and mechanical control.

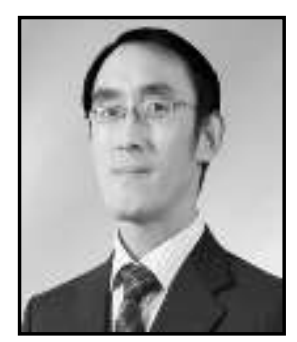

Yuegang Xing, He was born in 1974. He received the bachelor's degree from Nanjing University of Traditional Chinese Medicine in 1997.Currently, he is an associate chief physician in Huaiyin Hospital of Huaian City, China. His research interests include spinal joint disease and orthopedic surgery.

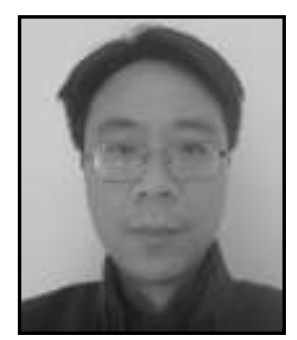

Xiaobing Chen, He was born in 1971. He received the Ph.D. degree from Nanjing University of Aeronautics and Astronautics in 2012. Currently, he is a professor in Huaiyin Institute of Technology, China. His research interests include reverse engineering and digital geometry processing. 